\title{
Early Results of Chimney Technique for Type B Aortic Dissections Extending to the Aortic Arch
}

\author{
Chen Huang ${ }^{1}$ Hanfei Tang ${ }^{2}$ Tong Qiao ${ }^{2}$ Changjian Liu $^{2}$. \\ Min Zhou ${ }^{2}$
}

Received: 28 January 2015/ Accepted: 19 May 2015/Published online: 12 June 2015

(C) Springer Science+Business Media New York and the Cardiovascular and Interventional Radiological Society of Europe (CIRSE) 2015

\begin{abstract}
Objective To summarize our early experience gained from the chimney technique for type B aortic dissection (TBAD) extending to the aortic arch and to evaluate the aortic remodeling in the follow-up period.

Methods From September 2011 to July 2014, 27 consecutive TBAD patients without adequate proximal landing zones were retrograde analyzed. Chimney stent-grafts were deployed parallel to the main endografts to reserve flow to branch vessels while extending the landing zones. In the follow-up period, aortic remodeling was observed with computed tomography angiography.

Results The technical success rate was $100 \%$, and endografts were deployed in zone $0(n=3,11.1 \%)$, zone $1(n=18,66.7 \%)$, and zone $2(n=6,22.2 \%)$. Immediately, proximal endoleaks were detected in 5 patients $(18.5 \%)$. During a mean follow-up period of 17.6 months, computed tomography angiography showed all the aortic stent-grafts and chimney grafts to be patent. Favorable remodeling was observed at the level of maximum descending aorta and left subclavian artery with expansion of true lumen (from $18.4 \pm 4.8$ to $25 \pm 0.86 \mathrm{~mm}$, $p<0.001$ and $27.1 \pm 0.62$ to $28.5 \pm 0.37 \mathrm{~mm}$, $p<0.001$ ) and depressurization of false lumen (from $23.7 \pm 2.7$ to $8.7 \pm 3.8 \mathrm{~mm}, p<0.001$, from $5.3 \pm 1.2$ to
\end{abstract}

Min Zhou

813477618@qq.com

1 Department of General Surgery, Affiliated Hospital of Nantong University, Nantong 226001, People's Republic of China

2 Department of Vascular Surgery, Nanjing Drum Tower Hospital, The Affiliated Hospital of Nanjing University Medical School, Nanjing 210008, People's Republic of China
$2.1 \pm 2.1 \mathrm{~mm}, p<0.001)$. While at the level of maximum abdominal aorta, suboptimal remodeling of the total aorta (from $24.1 \pm 0.4$ to $23.6 \pm 1.5 \mathrm{~mm}, p=0.06$ ) and true lumen (from $13.8 \pm 0.6$ to $14.5 \pm 0.4 \mathrm{~mm}, p=0.08$ ) was observed.

Conclusion Based on our limited experience, the chimney technique with thoracic endovascular repair is demonstrated to be promising for TBAD extending to the arch with favorable aortic remodeling.

Keywords Type B aortic dissection - Aortic arch · Endovascular repair · Chimney technique $\cdot$ Aortic remodeling

\section{Introduction}

For the descending aortic pathology, the thoracic endovascular aortic repair (TEVAR) has demonstrated favorable short- and mid-term results [1]. Endovascular management of the type $\mathrm{B}$ aortic dissection (TBAD) extending to the aortic arch remains challenging because of the involvement of supra-aortic branches. Although hybrid approaches have been proposed as alternatives, the mortality and mobility rates still remain high [2]. Other modified techniques such as fenestrated grafts or branch grafts were limited by the evolution of the treatment modalities and the complex procedures. [3-6] Another option to perfuse supra-aortic vessels is the chimney technique, firstly described by Greenberg et al. as a mean to rescue renal arteries [7]. Advantageously, this technique makes use of commercially available devices for planned surgeries or accidental coverage of the supra-aortic branches in emergent cases.

The chimney technique has been applied and presented promising outcomes to extend the proximal landing zone 
during the endovascular repair of thoracic aortic aneurysms [8-11]. However, in aortic dissections, this technique might be substantially different from that in aneurysm repairs owing to anatomical and pathophysiologic differences. Regarding the application of chimney grafts in aortic dissection, reports have been limited to small series with relatively short follow-up [12-15], and no study has focused specially on aortic remodeling with this technique. In this study, we summarize our initial experience with the chimney technique and evaluate the follow-up aortic remodeling for TBAD extending to the aortic arch.

\section{Method}

From September 2011 to July 2014, 27 patients with TBAD extending to the aortic arch underwent TEVAR combined with chimney technique, owing to the absence of sufficient proximal landing zone $(<15 \mathrm{~mm}$ landing zone distal to left subclavian artery). All the patients were assessed by a cardiac surgeon and considered unsuitable for traditional open surgery according to the ASA (American Society of Anesthesiologists). Characteristics of the patients are listed in Table 1. Informed signed consent was obtained from all patients involved, and this study was approved by the Institution Review Board.

Among these 27 patients, 25 were acute $(<15$ days) and 2 chronic ( $>92$ days). Seven were treated for malperfusion, three for rupture, 13 failure of best medical management, and four aneurismal enlargement. In addition, CTA was used to classify locations of the ET into greater curvature, lesser curvature, and anterior/posterior segments.

All procedures were performed under general anesthesia in hybrid operation room. Chimney stent-grafts for innominate artery (IA) and left common carotid artery (LCCA) were routinely applied if the thoracic stent-grafts were landed proximally in zone $0(n=3)$ and zone 1 $(n=18)$. In these cases, chimney grafts for left subclavian artery (LSA) were not applied with a view to avoiding

Table 1 Patient demographics

\begin{tabular}{ll}
\hline Number of patients & \\
\hline Age, year & $73.5(56-83)$ \\
Men & $19(70.4 \%)$ \\
Acute & $25(92.3 \%)$ \\
Hypertension & $18(66.7 \%)$ \\
Diabetes mellitus & $1(3.7 \%)$ \\
Hyperlipidemia & $2(7.4 \%)$ \\
Renal insufficiency & $1(3.7 \%)$ \\
COPD & $2(7.4 \%)$ \\
\hline
\end{tabular}

endoleak. Adjunctive LCCA-LSA bypass prior to endovascular repair was needed when these patients had a dominant left vertebral artery $(n=3)$. In other patients $(n=18)$, we intentionally overstented the LSA since the risk of arm ischemia, stroke, and paraplegia was relatively low. When the thoracic stent-grafts were landed proximally in zone 2, single-chimney stent-grafts for LSA $(n=6)$ were implemented.

After exposing the right common femoral artery (RCFA) through a small incision, catheters were inserted via the RCFA puncture under the guidance of appropriate wires. The aortic lesions were measured through angiography, and the diameters of aortic endograft and chimney graft were generally oversized by $10 \%$ aiming at adequate sealing of ET. In order to prevent stent-graft-induced distal dissections, distal restrictive bare stents (Sinus, OptiMed, Germany) were used when the estimated mismatch rate (ratio of distal diameter of stent-graft to long diameter of true lumen) was greater than $120 \%$.

Guidewires for chimney grafts were placed via left brachial artery access, left common carotid artery, or right common carotid artery access. Percutaneous access guided by ultrasound was used for small sheaths $(\leq 6-F)$, and a cutdown was needed for $>6-\mathrm{F}$ sheaths to avoid iatrogenic vascular injury. The relative positions of super-stiff wire for aortic endograft and guidewire for chimney graft were adjusted in order to avoid endoleak. For ET located in the posterior segment, the chimney graft should be deployed in front of the aortic stent-graft (left-anterior oblique view), close to the anterior wall of thoracic aorta, whereas for ET located in anterior segment, the chimney graft deployed behind the aortic stent-graft (Fig. 1). In most cases, ET was located in the greater curvature or lesser curvature, where the position of chimney graft was not necessarily adjusted. Chimney grafts [Fluency (C.R. Bard, USA); or Viabahn (GORE, USA); E-LUMINEXX (C.R. BARD, USA)] were then advanced to the aortic arch through the guidewires. The aortic endograft was introduced upward to the thoracic aorta, and types of endografts included TAG (W.L. Gore, $n=9$ ), Valiant (Medtronic, USA; $n=9$ ), and Hercules (MicroPort, China, $n=9$ ). 1-2-cm overlap between the aortic endograft and chimney graft was recommended. Under controlled hypotension (systolic blood pressure $<90 \mathrm{mmHg}$ ) and fluoroscopy, the aortic endografts were deployed, followed by the rapid deployment of the chimney graft parallel to the main aortic stent-graft, except in the IA chimney cases. In those cases, considering the reduction of cerebral blood flow after aortic endograft deployment, the chimney grafts for IA and LCCA were deployed firstly followed by the aortic stent-graft. The chimney graft was then dilated with suitable angioplasty balloon. 

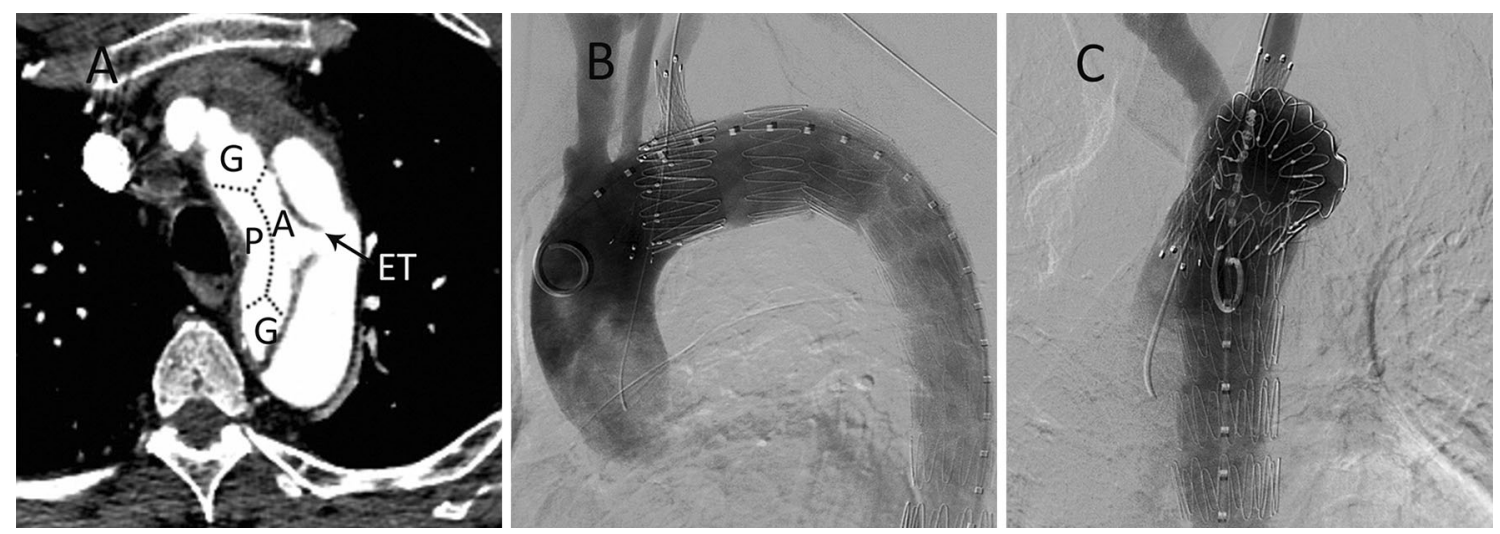

Fig. 1 The gutter should be formed as far away from ET as possible. A Pre-operative computed tomography angiography (CTA) showed a TBAD patient with ET located at the anterior segments, and the entry tear (ET) was adjacent to the LSA $(\leq 15 \mathrm{~mm})$. The arrow indicated the position of the ET. B Left-anterior oblique view and $\mathbf{C}$ ortho position of intra-operative digital subtraction angiography (DSA)

Completion ascending aortic angiogram was performed, and proximal fixation of the endograft, immediate endoleak, and revascularization of supra-aortic branches were evaluated. Finally, the RCFA access site was closed with a 5-0 Prolene. Technical success was defined as the accurate proximal deployment of the aortic endograft and preserved supra-aortic branches. All patients were routinely administered with oral aspirin (100 mg for at least 6 months). Table 2 gives the characteristics of all procedures.

Follow-up: The follow-up protocol included computed tomography angiography (CTA) performed after 3, 6, and demonstrated the completed exclusion of the ET. C Since the ET was located at the anterior segments, the chimney graft was then deployed close to the posterior wall of thoracic aorta in order to avoid endoleak. $A$ the anterior segments, $P$ the posterior segments, $G$ the greater curvature

12 months and yearly thereafter. Three-dimensional reconstructions of the $\mathrm{CT}$ scans were used for measuring the lumen diameters and the true and overall aortic lumen diameters of the latest CTA at the level of the LSA ostium, the MDA point, and the MAA level.

\section{Statistical Analysis}

Continuous variables were represented as the mean \pm standard deviation, whereas categorical and ordinal data were summarized as frequencies and percentages. The
Table 2 TEVAR

characteristics

\begin{tabular}{ll}
\hline & Mean \pm SD or $n(\%)$ \\
\hline Acute/chronic & $25 / 2$ \\
Proximal landing zone(zone 0/zone 1/zone 2) & $3 / 18 / 6$ \\
Locations of ET (L/G/A/P) & $4 / 16 / 3 / 4$ \\
Two chimney stents & $3(11.1 \%)$ \\
Stent types of chimney grafts (covered/bare) & $22 / 8$ \\
Diameter of chimney grafts (mm) & $8.9 \pm 1.4$ \\
Length of chimney grafts (mm) & $58.7 \pm 16.6$ \\
Diameter of aortic stent-grafts (mm) & $36.4 \pm 2.8$ \\
Length of aortic stent-grafts (mm) & $166.7 \pm 24.0$ \\
LCCA-LSA bypass & $3(11.1 \%)$ \\
Post-operative endoleak (Type I/Type II) & $4 / 1$ \\
Follow-up time (month) & $17.6 \pm 9.6$ \\
Follow-up results & \\
Patent & $27(100 \%)$ \\
Endoleak resolved & $2(40 \%)$ \\
Endoleak diminished & $2(40 \%)$ \\
Endoleak unchanged & $1(20 \%)$ \\
\hline Continuous
\end{tabular}

Continuous data are presented as the mean \pm standard deviation; categorical data are given as the counts. $E T$ entry tear, $L$ the lesser curvature, $G$ the greater curvature, $A$ the anterior segments, $P$ the posterior segments 

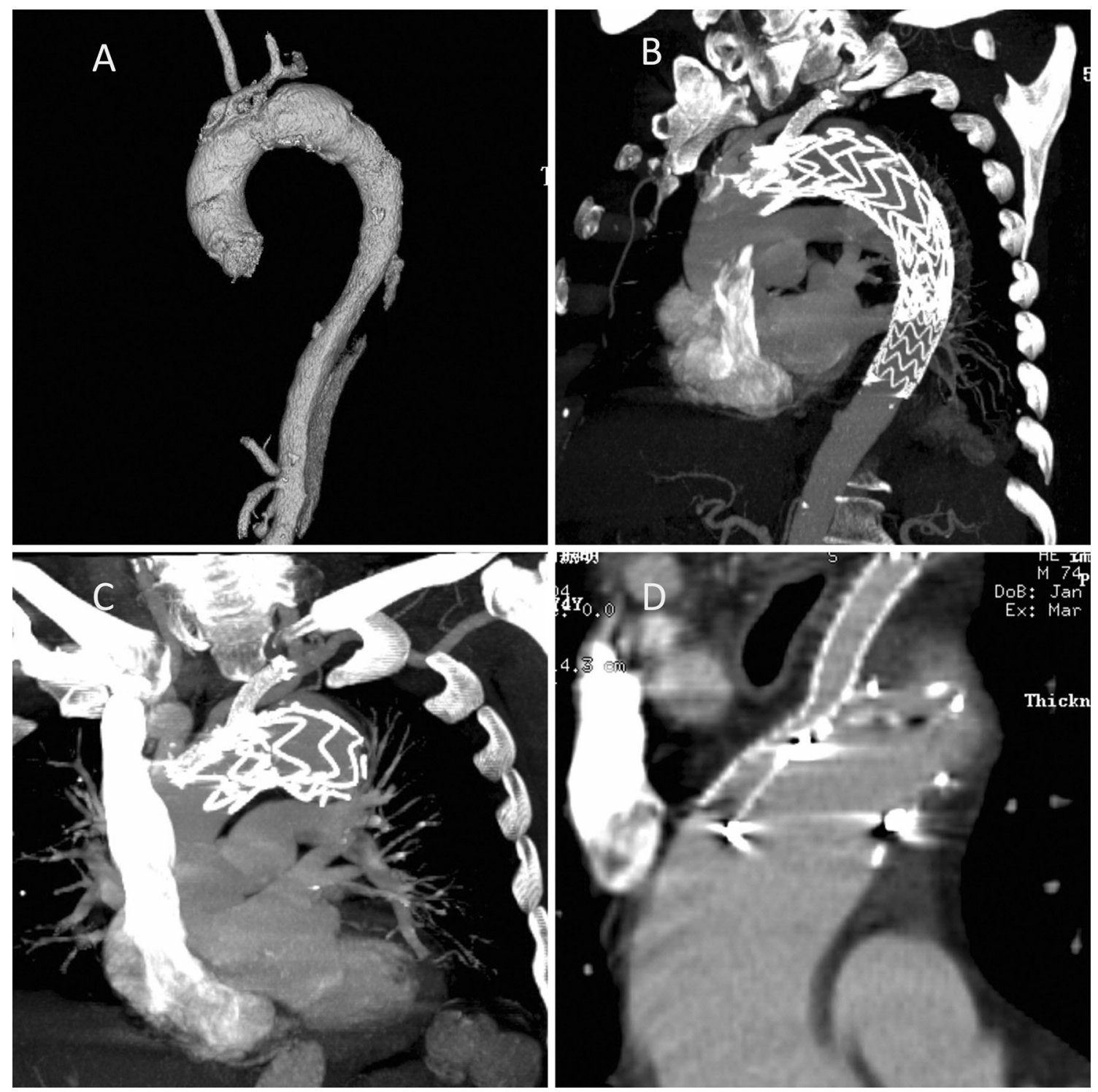

Fig. 2 Left subclavian artery (LSA) chimney stenting in TEVAR for TBAD. A Pre-operative computed tomography angiography (CTA) showed type B aortic dissection with tear adjacent to the LSA. B-D Follow-up CTA showed exclusion of the tear and a patent chimney stent in the LSA

comparisons of pre- and post-operative values were drawn by a paired $t$ test, and significant differences were assumed at $p<0.05$. Statistical analyses were performed using software (SPSS Inc., Chicago, Illinois).

\section{Results}

The procedure of all 27 patients was technically successful. The aortic stent-grafts were deployed in Zone 2 (six patients, Fig. 2), Zone 1 (18 patients, Fig. 3), and Zone 0 (three patients) according to Ishimaru classification [13]. Distal restrictive bare stents were used in 18 cases with an estimated mismatch rate greater than $120 \%$. Totally 30 chimney grafts were used, of which 22 were covered stents and 8 were bare stents. Chimney-graft compression by the main aortic stent-graft was not observed during the procedure. Immediately proximal endoleaks were detected in five patients $(18.5 \%)$ despite that the aggressive angioplasties were performed, and for most of them the ET was located at the greater curvature (four patients), and another one patient with endoleak had ET at the anterior segment. No endoleaks were detected in patients with ET located at the lesser curvature and the posterior segment. There was no perioperative death or acute spinal cord ischemia (SCI), but two minor stokes were documented. These two patients suffered from neurological deficit of limbs and recovered completely after $2-3$ weeks. 

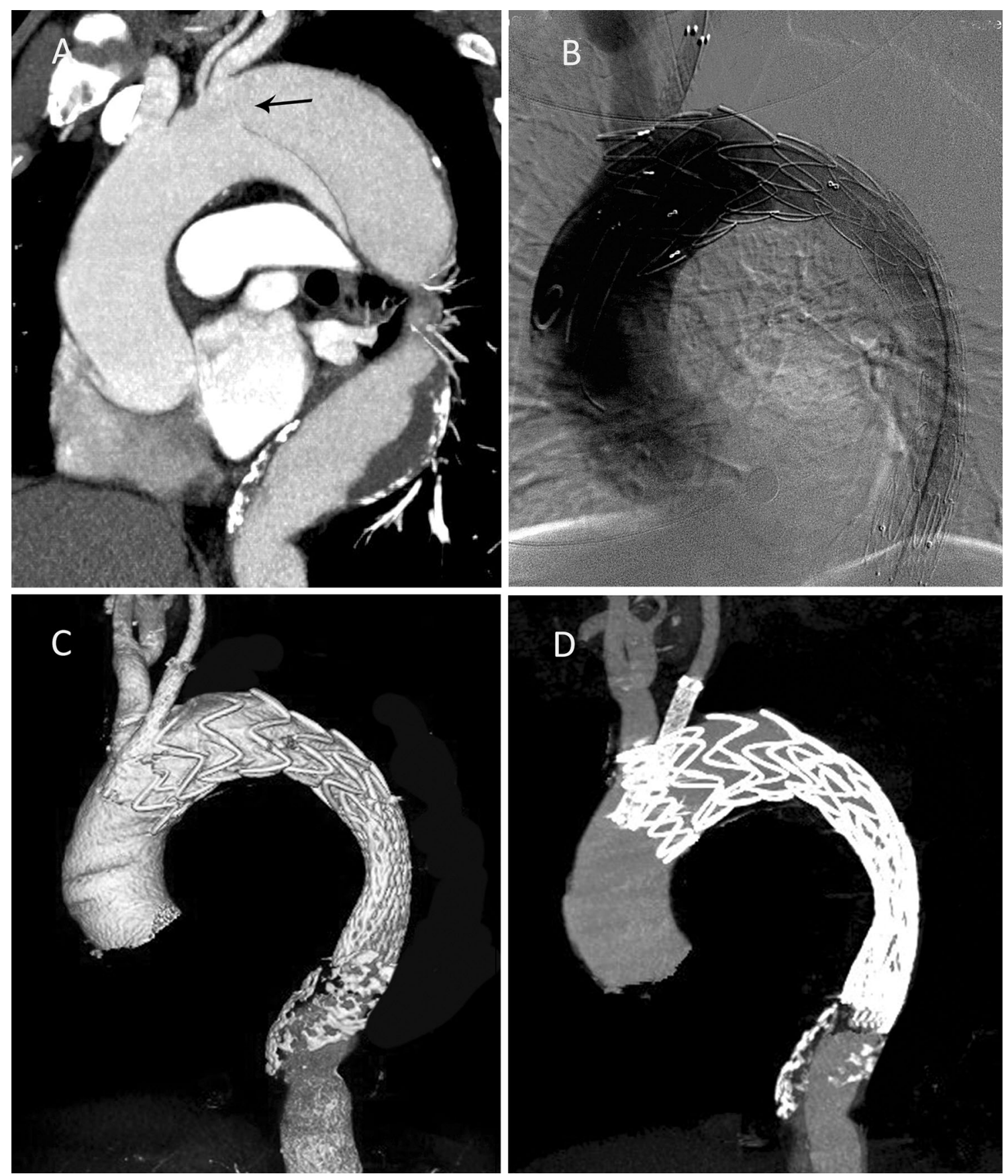

Fig. 3 A Pre-operative computed tomography angiography (CTA) showed a patient with entry tear (ET) adjacent to the left common carotid artery (LCCA). The arrow indicated the position of the ET. B Digital subtraction angiography (DSA) demonstrated complete

The mean follow-up was 17.6 months (range 3-34 months), during which no death was aneurysm related. One patient died of myocardial infarction 21 months later. CTA showed all the aortic stent-graft and chimney grafts to be patent, with no migration and fracture. Among five cases with immediate proximal endoleak, two resolved spontaneously (3 months after operation), two diminished (6 and 9 months after

exclusion of the ET and preserved LCCA blood flow. C and D Preoperative CT scan showed patency of the aortic stent-graft and the chimney graft for LCCA, compression of the false lumen and enlargement of the true lumen

operation, respectively), and one was stable. Three persistent endoleaks were not treated because sac enlargement was not detected during the follow-up period, and these patients remain being under close surveillance. Complete thrombosis of the false lumen of the aorta was noted in 16 patients (16 of 27 , $59.2 \%$ ), and partial thrombosis was noted in 11 patients (11 of $40.7 \%)$. 
Mean overall aortic lumen diameter at the point of MDA significantly decreased from $42.1 \pm 3.8 \mathrm{~mm}$ before the stent grafting to $33.7 \pm 3 \mathrm{~mm}$ during the follow-up $(p<0.001)$. Furthermore, the diameter at the level of LSA decreased significantly, from $32.4 \pm 1.4$ to $30.6 \pm 2.0 \mathrm{~mm}$ $(p=0.002)$. MAA $(24.1 \pm 0.4$ vs. $23.6 \pm 1.5 \mathrm{~mm}$, $p=0.06)$ was not significantly different between pre-operative and follow-up measurements. True lumen diameter increased from $18.4 \pm 4.8$ to $25 \pm 0.86 \mathrm{~mm}(p<0.001)$ at the point of MDA, from $27.1 \pm 0.62$ to $28.5 \pm 0.37 \mathrm{~mm}$ $(p<0.001)$ at the level of the LSA, and from $13.8 \pm 0.6$ to $14.5 \pm 0.4 \mathrm{~mm}$, but without significant difference $(p=0.08)$, at the level of MAA. The diameter of false lumen at different levels significantly decreased in the follow-up: from $23.7 \pm 2.7$ to $8.7 \pm 3.8 \mathrm{~mm}(p<0.001)$ at the point of MDA, from $5.3 \pm 1.2$ to $2.1 \pm 2.1 \mathrm{~mm}$ $(p<0.001)$ at the level of the LSA, and from $10.3 \pm 2.4$ to $9.1 \pm 3.3 \mathrm{~mm}(p=0.003)$ at the level of MAA (Fig. 4).

\section{Discussion}

The chimney technique represents a viable option for treating TBAD patients involving supra-aortic branches. This technique, firstly performed in 2002, was thought to be a "bailout" option to rescue inadvertently covered vessels, and the follow-up observation proved the good results of this alternative $[14,16]$. This technique was then used even in planned procedures for patients considered unsuitable for open repair [12, 17]. Its relatively easy procedure, familiar to most practicing surgeons, has shown its potential advantages and has been reported by increasingly more literature [3-6]. Nevertheless, scarce studies focus on safety and efficacy of chimney technique in TBAD. In the treatment of aortic dissection, some noteworthy details should be paid attention to for good outcomes.

The first choice for patients with TBAD extending to the aortic arch is still controversial. The repair hereof is more challenging compared to classical TBAD, for which open surgery has been a feasible and safe approach, with acceptable results, especially in centers of excellence. Early results of chimney technique just demonstrated the feasibility only. Owning to the limited quantity of reports and the lack of long-term observation, some surgeons suggested that chimney technique should be considered only in acute poor surgical risk patients or as a bailout in case of unintentional coverage of supra-aortic vessels. However, one has to take the learning curve into account for developing stent-grafts and chimney technique procedure. In addition, considering the relatively high mortality among patients treated by open surgery, a niche may
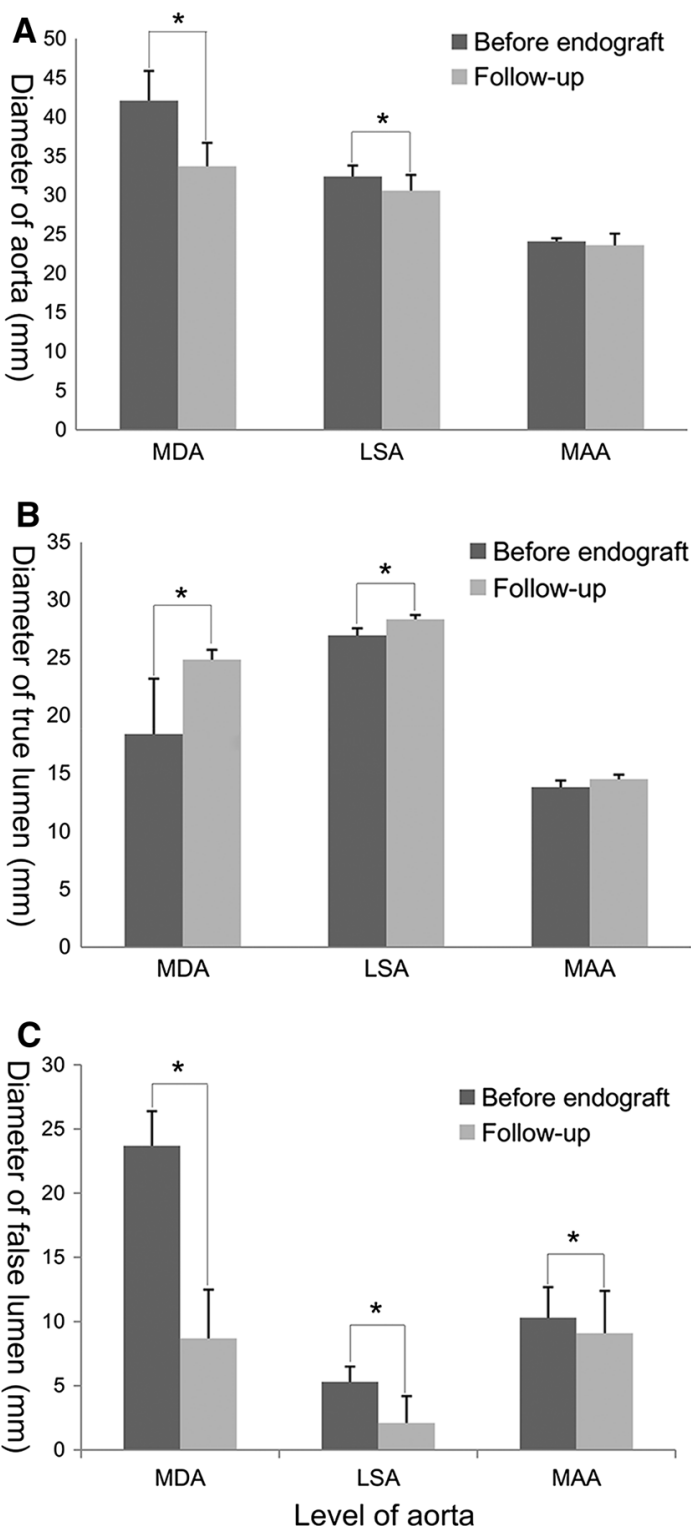

Fig. 4 Bar graphs illustrating the mean diameter of $\mathbf{A}$ the total diameter, $\mathbf{B}$ the true lumen, and $\mathbf{C}$ the false lumen compared between pre-operative and follow-up measurements. The asterisk indicates that the change was significant $(p<0.05)$. MDA maximum descending aorta, $L S A$ left subclavian artery, MAA maximum abdominal aorta

therefore exist for TEVAR to supplement open surgery for patients with TBAD extending to the arch.

High technical success, low complication, and excellent post-operative patency rate engendered enthusiasm for chimney technique. In this study, the procedure of all 27 patients was technically successful, similar to previous studies [12]. In three cases, the aortic endograft had to be deployed into Zone 0 to obtain an adequate proximal landing zone. This procedure was associated with considerable risks since IA and LCCA serve as branch vessels for the cerebral circulation. The innominate stent-graft should 
be initially deployed to preserve RCCA flow to prevent cerebrovascular events. Notably, two patients in our series had documented stroke. It is likely that the required manipulation of supra-aortic vessels was the source of emboli in our cohort. Some researchers believed that the likelihood of cerebral embolization was associated with the implantation of aortic endograft into atherosclerotic lesions [15]. During a mean follow-up of 17.6 months, all the chimney graft remained to be patent. Routine administration of aspirin is one of the factors possibly contributing to the patency of the chimney grafts, and chimney graft for the supra-aortic branches might be of higher blood flow in contrast to the chimney grafts for visceral arteries. In our opinion, another speculative reason for the high patency rate was the relatively short follow-up period. Chimney stent thrombosis may occur at a higher rate with increasing follow-up time. The chimney graft is usually under great mechanical pressure from the aortic endograft, and the chimney graft can lead to alternation of branch vessel anatomy (e.g., take-off angle of the branch vessel), so obstruction or fracture of the chimney grafts could occur anytime during the follow-up period. Aggressive image surveillance protocol is mandatory despite the early positive outcome of our study.

Endoleaks, especially type I endoleak, are always considered as the Achilles' heel of the chimney technique, and they were poorly understood in TBAD. TBAD is characterized by primary ETs which might locate at the greater curvature, lesser curvature, and anterior or posterior segments. The gutters between the main aortic and chimney graft become the potential channel between ET and the aortic canal. As a result, the gutter should be formed as far away from ET as possible. We observed its consistence with prior studies that the immediate endoleak rate was higher especially when the ET was located at the greater curvature of the aortic arch [17]. Theoretically, the supraaortic branches took off from the greater curvature, and it was hard to keep the gutter away from ET located at the greater curvature. As a result, the relative positions of ET and chimney grafts are of great importance to avoid endoleak. When ET was located in posterior segment, the chimney graft should be deployed in front of the aortic stent-graft, close to the anterior wall of thoracic aorta, whereas chimney graft deployed behind the aortic stentgrafts when ET was located in anterior segment.

Patients with immediate type I endoleak were usually treated with kissing balloon technique to minimize gutter formation. The safety of this approach needed further exploration considering the highly fragile aortic wall in the aortic dissection. Excessive angioplasties might cause retrograde type A dissection in aortic dissection. Another promising technique to deal with endoleak is the minimally invasive coil embolization. The gutters could be selectively catheterized and embolized with coils [18]. Anyway, it was encouraging that most of the endoleaks resolved ( $40 \%$ ) or diminished $(40 \%)$ during the follow-up period.

Choosing the ideal configuration for chimney graft is another controversial topic. Both covered and uncovered stents have been used in the chimney technique. In our experience, we preferred using covered stent. Covered stent might be helpful for reducing the risk of endoleak since it can decrease the blood flow coming via the meshes of bare stent into the gutter. Moreover, the gutter is like a blind channel, where thrombosis could be formed and be a cause of cerebral emboli, and covered stents might reduce this risk [19]. However, the large delivery system is the main drawback of the covered stent. Some of the covered stents had to be implanted by surgical access, whereas the bare stent can be implanted by percutaneous access. As a result, some surgeons still prefer using bare stents, and the reasonable choice of different stents needs to be confirmed by future studies.

Successful TEVAR is characterized by effective aortic remodeling with depressurization of false lumen and expansion of true lumen, so it is in the TEVAR in combination with chimney technique. During the follow-up observations, favorable remodeling was observed in several aortic levels containing the level of MDA and LSA, but suboptimal remodeling of the aorta was noticed at the level of MAA. The discrepancy of remodeling between different aortic levels may be attributed to residual distal fenestrations located in the abdominal aorta. Hughes GC [20] found that distal aorta with fewer than three residual fenestrations was the ideal anatomy for complete aortic remodeling. As a result, while chimney technique makes it possible to cover the proximal ET, care should be taken to deal with the distal entry aiming at favorable aortic remodeling.

It is one of our acknowledgements that the present study is limited. Our current experience to avoid endoleak is to form the gutter as far away from ET as possible. However, this hypothesis could not be confirmed without rigorous comparative effectiveness data in this preliminary study. Furthermore, long-term follow-up observation and comparison to fenestrated endovascular repair or open repair were needed to determine the long-term efficiency of the chimney technique.

\section{Conclusion}

Our limited experience demonstrated that the chimney technique with TEVAR seems promising for TBAD extending to the arch with favorable aortic remodeling. It could be completed with a high technical success rate, and its post-operative complications were acceptable. 
Considering the pathophysiologic characteristics of aortic dissection, the gutter should be formed as far away from ET as possible for avoiding endoleak.

Conflict of interest The authors Chen Huang, Hanfei Tang, Tong Qiao, Changjian Liu, and Min Zhou have no conflicts of interest to declare.

Statement of Informed consent Additional informed consent was obtained from all individual participants for whom identifying information is included in this article.

\section{References}

1. Bavaria JE, Appoo JJ, Makaroun MS, Verter J, Yu ZF, Mitchell RS, et al. Endovascular stent grafting versus open surgical repair of descending thoracic aortic aneurysms in low-risk patients: a multicenter comparative trial. J Thorac Cardiovasc Surg. 2007;133:369-77.

2. Hiraoka A, Chikazawa G, Tamura K, Totsugawa T, Sakaguchi T, Yoshitaka H. Clinical outcomes of different approaches to aortic arch disease. J Vasc Surg. 2015;61(1):88-95.

3. Yuan L, Feng X, Jing Z. Endovascular repair of a thoracic arch aneurysm with a fenestrated stent-graft. $\mathrm{J}$ Endovasc Ther. 2008;15:539-43.

4. Murphy EH, Stanley GA, Ilves M, Knowles M, Dimaio JM, Jessen ME, et al. Thoracic endovascular repair (TEVAR) in the management of aortic arch pathology. Ann Vasc Surg. 2012;26:55-66.

5. Monahan TS, Schneider DB. Fenestrated and branched endografts for repair of complex aortic aneurysms. Semin Vasc Surg. 2009;22:132-9.

6. Yoshida RA, Kolvenbach R, Yoshida WB, Wassijew S, Schwierz E, Lin F. Total endovascular debranching of the aortic arch. Eur J Vasc Endovasc Surg. 2011;42:627-30.

7. Greenberg RK, Clair D, Srivastava S, Bhandari G, Turc A, Hampton J, et al. Should patients with challenging anatomy be offered endovascular aneurysm repair? J Vasc Surg. 2003;38(5): 990-6.

8. Shahverdyan R, Gawenda M, Brunkwall J. Triple-barrel graft as a novel strategy to preserve supra-aortic branches in arch-TEVAR procedures: clinical study and systematic review. Eur J Vasc Endovasc Surg. 2013;45(1):28-35.
9. Lachat M, Veith FJ, Pfammatter T, Glenck M, Bettex D, Mayer $\mathrm{D}$, et al. Chimney and periscope grafts observed over 2 years after their use to revascularize 169 renovisceral branches in 77 patients with complex aortic aneurysms. J Endovasc Ther. 2013;20(5):597-605.

10. Samura M, Zempo N, Ikeda Y, Hidaka M, Kaneda Y, Suzuki K, et al. Endovascular repair of distal arch aneurysm with doublechimney technique. Ann Thorac Surg. 2013;95(5):1778-80.

11. Pecoraro F, Pfammatter T, Mayer D, Frauenfelder T, Papadimitriou D, Hechelhammer L, et al. Multiple periscope and chimney grafts to treat ruptured thoracoabdominal and pararenal aortic aneurysms. J Endovasc Ther. 2011;18(5):642-9.

12. Zhu Y, Guo W, Liu X, Jia X, Xiong J, Wang L. The single-centre experience of the supra-arch chimney technique in endovascular repair of type B aortic dissections. Eur J Vasc Endovasc Surg. 2013;45(6):633-8.

13. Mitchell RS, Ishimaru S, Ehrlich MP, Iwase T, Lauterjung L, Shimono T, et al. First international summit on thoracic aortic endografting: roundtable on thoracic aortic dissection as an indication for endografting. J Endovasc Ther. 2002;9:II98-105.

14. Mangialardi Nicola, Serrao Eugenia, Kasemi Holta. Alberti V, Fazzini S, Ronchey S. Chimney Technique for Aortic Arch Pathologies: an 11-Year Single-Center Experience. J Endovasc Ther. 2014;21:312-23.

15. Samura M, Zempo N, Ikeda Y, Hidaka M, Kaneda Y, Suzuki K, et al. Endovascular repair of distal arch aneurysm with doublechimney technique. Ann Thorac Surg. 2013;95(5):1778-80.

16. Criado FJ, Barnatan MF, Rizk Y, Clark NS, Wang CF. Technical strategies to expand stent-graft applicability in the aortic arch and proximal descending thoracic aorta. J Endovasc Ther. 2002;9 (suppl II):II32-8.

17. Xue Y, Sun L, Zheng J, Huang X, Guo X, Li T, et al. The chimney technique for preserving the left subclavian artery in thoracic endovascular aortic repair. Eur J Cardiothorac Surg. 2015;47(4):623-9.

18. Sugiura K, Sonesson B, Akesson M, Björses K, Holst J, Malina M. The applicability of chimney grafts in the aortic arch. J Cardiovasc Surg (Torino). 2009;50(4):475-81.

19. Baldwin ZK, Chuter TA, Hiramoto JS, Reilly LM, Schneider DB. Double-barrel technique for endovascular exclusion of an aortic arch aneurysm without sternotomy. J Endovasc Ther. 2008;15: $161-5$.

20. Hughes GC, Ganapathi AM, Keenan JE, Englum BR, Hanna JM, Schechter MA, et al. Thoracic endovascular aortic repair for chronic DeBakey IIIb aortic dissection. Ann Thorac Surg. 2014;98(6):2092-7. 